\title{
Level of Resident Care Need and Staffing by Size of Nursing Home under the Public Long-term Care Insurance in South Korea
}

\author{
Bae, Sung-Heui ${ }^{1} \cdot \mathrm{Kim}_{\text {, Hongsoo }}^{2}$ \\ ${ }^{1}$ Associate Professor, College of Nursing, Ewha Womans University, Seoul \\ ${ }^{2}$ Professor, Graduate School of Public Health, Department of Public Health Sciences; Institute of Aging; Institute of Health and Environment, \\ Seoul National University, Seoul, Korea
}

\begin{abstract}
Purpose: Formal long-term care in nursing homes became a popular means of caring for frail elders in South Korea. This universal long-term care (LTC) insurance for elders was introduced in 2008. The aim of this study was to provide information on the extent of and variations in residents' care needs and nursing staffing characteristics across nursing homes. Methods: This was a population-based secondary analysis of a large national survey study involving 1,472 older residents in 92 nursing homes in South Korea in 2013. Characteristics of residents and nursing homes were collected by functional assessment and an examination of administrator surveys for nursing home size was done using descriptive analysis. Results: Small and small-medium nursing homes were more likely to provide care to non-Medical-aid beneficiaries and older people with lower care need level. These smaller homes also better met the minimum nursing staffing standard. However, they were less likely to hire registered nurses (RNs), as it is not yet a staffing requirement for nursing homes in Korea. Conclusion: South Korea has a policy agenda to increase nursing staffing level, especially by adding RNs, and decrease variations in staffing levels by nursing home size. Further evidence on care-need level, proper resource allocation, and policies designed to strengthen the LTC workforce is essential to advance the LTC system in Korea.
\end{abstract}

Key Words: Nursing homes; Personnel staffing and scheduling; Needs assessment; Long-term care insurance

\section{INTRODUCTION}

Population aging is a global phenomenon. The population of every country in the world is expected to have a substantial increase in people aged 65 or older by 2030 [1]. By 2030, the number of older persons will be larger than the number of children aged $0 \sim 9$ years (1.4 billion vs. 1.3 billion) [1]. The speed of population aging is much faster in East Asia than in Western countries. South Korea is one of the most rapidly aging countries in the world. It took only 18 years for the population aged $65+$ to transition from $7 \%$ to $14 \%$ of the population in South Korea compared to 69 years in the United States and 115 years in France [2]. Living longer with chronic conditions increases long-term care needs. Sociocultural changes such as an increase in women's labor participation and nuclear families have resulted in a heavy burden of family care for older persons.

The introduction of public long-term care insurance (LTCI) for elders in South Korea in July 2008 opened a new era in the provision of formal long-term care services [3]. People aged 65 or older with a certain level of functional dependency are eligible for the LTCI. Older adults compose about $13.3 \%$ of the Korean population [3]. Of these older adults, about $7.5 \%$ have severe functional limitations [3]; they receive LTC services under the public LTCI. The number of people who receive LTC services is expected to increase [3]. The Korean government has made an effort to build an infrastructure for LTCI. The number of nursing homes rapidly increased from 4,326 in

Corresponding author: Kim, Hongsoo https://orcid.org/0000-0002-5539-7653

Department of Public Health Sciences, Seoul National University, 1 Gwanak-ro, Gwanak-gu, Seoul 08826, Korea.

Tel: +82-2-880-2723, Fax: +82-2-762-9105, E-mail: hk65@snu.ac.kr

Received: Sep 30, 2019 / Revised: Dec 3, 2019 / Accepted: Jan 8, 2020

This is an open access article distributed under the terms of the Creative Commons Attribution Non-Commercial License (http://creativecommons.org/licenses/ by-nc/3.0), which permits unrestricted non-commercial use, distribution, and reproduction in any medium, provided the original work is properly cited. 
2012 to 5,187 in 2016 [3].

Nursing home services under the public LTCI, which offers comprehensive universal coverage in Korea, have several distinctive features compared to other countries. Regardless of income level, all Korean older adults with a certain level of functional limitation based on nationally standardized care-needs assessment system are eligible for the LTCI [3]. Unlike nursing homes in the U.S., nursing homes in Korea are mainly social care organizations whose main service is to provide daily living assistance [4]. Under the current system, when residents in nursing homes have health or medical care needs, they are supposed to visit local clinics or be transferred to hospitals (acute or long-term care hospitals). All older Koreans who are beneficiaries of the public LTCI are also eligible for the National Health Insurance [3,4]. For this reason, the legal staffing requirement for nursing homes is different from that in other countries. The workforce in nursing homes mainly consists of direct care workers (personal care assistants) [5]. Specifically, according to Korea's Elderly Welfare Act 22, nursing homes are required to hire one nursing staff, either a registered nurse ( $\mathrm{RN}$ ) or a nurse aid (NA), per 25 residents [6]. For other care workers, the staffing requirement is one direct care worker per 2.5 residents and one social worker per 100 residents. All these staffing standards aim to meet resident care needs based on appropriate delivery systems for long-term care services, which are more likely to focus on social care services.

Regarding staffing regulations for nursing homes, several countries have adopted staffing standards. The main drivers for enacting such policies are quality concerns in nursing homes, such as resident weight loss, pressure ulcers, infections, and other preventable events [7]. In the U.S., federal staffing standards for all certified nursing homes require one $\mathrm{RN}$ for one shift $(8$ consecutive hours) 7 days a week. For the two remaining shifts, one $\mathrm{RN}$ and one licensed nurse (either an RN or a licensed vocational nurse/licensed practical nurse) are required [7]. Several states in the U.S. have also set higher licensed nurse requirements than the federal minimum. In Canada, provincial governments are responsible for setting staffing standards for Canadian nursing homes (residential care facilities). Most provinces require an RN on duty 24 hours a day, while several provinces (e.g. Alberta and Newfoundland) require nursing homes to provide a certain number of care hours per patient day of nursing [7]. In England, national standards have been established for residential care homes and nursing homes. Although actual staffing levels are not specified by the national standards, they do require nursing homes to specify staffing levels and staff qualifications based on the care needs of residents [7]. The staffing standards of all these countries focus on providing sufficient staff with the qualifications necessary to meet residents' care needs and ensure quality of care in nursing homes.

As mentioned above, while nursing homes in Korea were originally designed to be social care institutions for elders, a recent study found that older residents in Korean nursing homes had similar care needs to older people in LTC hospitals [4]. Older residents in nursing homes had several chronic diseases including hypertension, diabetes, and/or musculoskeletal diseases, and they had a great need for health care services [8]; this implies there may be a gap between nursing home staffing and resident care-needs in Korean nursing homes. Such a gap can lead to suboptimal quality of care in nursing homes. However, little is known about nursing home residents' characteristics, their care needs, or nursing home staffing. Therefore, the purpose of this study was to describe resident and nursing home characteristics and examine residents' care needs and nursing home staffing characteristics. Through investigating residents' care needs, the aim of this study was to provide important information to support staffing management based on care needs and to improve of the quality of care in Korean nursing homes. The specific objectives of the study were as follows:

- To compare resident and nursing home characteristics by size of nursing home.

- To compare residents' care needs and nursing home staffing characteristics by size of nursing home.

\section{METHODS}

\section{Study Design and Study Population}

This was a secondary analysis of a large national survey study on nursing homes under the public LTCI that was conducted in March through June 2013. The study was entitled "A study to advance health and long-term care service systems for older people through a nationwide survey" and was funded by the National Health Insurance Services [4]. The corresponding author had primary responsibility for the study design, data collection, and analyses of the parent survey study. A nationally representative sample of nursing homes was selected by a two-stage stratified random sampling approach using geographic region and facility size as strata. A random sampling of $20 \%$ of older residents in each home was then conducted using a resident roster. Nursing homes that 
were newly opened within one year and/or had a number of beds of nine or less were excluded because the resident profile and service provision of these nursing homes were likely to be different from their counterparts. Residents who had been admitted to a nursing home for less than one month were also excluded because newly admitted patients could have unstable conditions and/or care providers might not know those patients well. The final sample consisted of 1,472 residents aged 65 years or older in 92 nursing homes, representing about $4.4 \%$ of the target nursing home population. The sample size was calculated with a medium to large effect size (.40), .05 significance levels, and 8 degrees of freedom for $x^{2}(n=94)$ and 5 degrees of freedom for ANOVA $(n=80)$. The sample size was sufficient for data analysis. The sample nursing homes were similar to the national population of nursing homes in ownership mix, nursing staffing level, and spread of establishment years. This study was reviewed and approved by the Institutional Review Board of the institution where the corresponding author is affiliated (SNU IRB No. E1806/003-005).

\section{Instruments and Procedure}

In the parent study, the functional status and health conditions of older nursing home residents were examined using the international Resident Assessment Instrument for Long Term Care Facilities (interRAI LTCF), a comprehensive assessment system used internationally for research and practice purposes [9]. The inter RAI LTCF can measure the multi-dimensional functional status of institutionalized adults, including activities of daily living, comorbidities, services provided, and more. The Korean version of the interRAI LTCF was developed through a translation and back-translation procedure and psychometrically tested with 908 older adults in 27 longterm care institutions including 10 nursing homes [9]. The internal consistency of key scales in the Korean version of the interRAI LTCF, assessed by Cronbach's $\alpha$, was good $(\geq .75)$. The overall mean kappa statistics to assess inter-rater reliability of the items in the interRAI LTCF were .78. A detailed report on the reliability of the instrument can be found in Kim et al. [9]. In the parent study, older residents who agreed to participate in the study as themselves or by proxies only were assessed by staff nurses and social workers who provided care to these residents. All the assessors had to attend a training session consisting of lectures, a question and answer session, and a hands-on assessment exercise. Institutional-level variables were collected from nursing home administrators us- ing a survey form including nursing home characteristics such as number of beds and staffing levels. Helpdesk services were consistently provided during data collection via phone call and Short Message Service. These collected data were reviewed and corrected if any error or missing information was identified in the parent study.

\section{Variables}

Nursing home size. Nursing homes were divided into four groups: small (10 25 beds), small-medium (26 37 beds), medium (38 62 beds), and large (63 or more). According to Elderly Welfare Act 22 in Korea, nursing homes are required to hire one nursing staff (either RN or NA) per 25 residents. This act allows nursing homes with up to 37 beds $(37 / 25=1.48)$ to hire one nursing staff, although hiring only one nursing staff is actually understaffing. Thus, we categorized nursing homes into small (10 25 beds) and small-medium (26 37 beds) to distinguish these two homes by nurse staffing condition. Based on this law, nursing homes need to hire two nursing staff when they have 62 beds. Thus, we also categorized nursing homes into medium (nursing homes with 38 62 beds) and large (nursing homes with 63 or more).

Nursing home characteristics. Nursing home characteristics included ownership (private or public); occupancy rate (number of patients/total nursing home beds $\left.{ }^{*} 100\right)$, which was grouped into three; year of foundation (in 2008 [the year of LTCI inception] or between 2009 and 2011); and location (rural or urban).

Residential characteristics of the nursing home. Residential characteristics and care needs were assessed by age, gender, being a Medical-aid beneficiary (yes/no), living alone prior to admission, length of stay ( $\leq 692 \mathrm{vs}$. $>692$ days [median value]), diagnosis with stroke (yes/ no) or dementia (yes/no), activities of daily living (ADL) score $(0 \sim 2=$ independent to limited impairment; $3 \sim 4=$ extensive assistance required; $5 \sim 6=$ dependent or total dependence); cognition levels $(0 \sim 2=$ intact to mild impairment; $3 \sim 4=$ moderate or severe impairment; $5 \sim 6=$ severe or very severe impairment); depression condition ( $0=$ normal; 1 2=low risk; 3 14=high risk); and care-need levels (grade 1, grade 2, grades $3 \& 4$ ). To measure ADL, the 4 -item Activities of Daily Living Hierarchy Scale $[4,10]$ was used. To measure cognition, the 4-item Cognitive Performance Scale [11] related to decision-making, short-term memory, being understood by others, and eating was used. Depression was measured by the 7-item Depression Rating Scale [4,12]. A level of care-need is assigned to each LTCI beneficiary every $1 \sim 2$ years based on a na- 
tion-wide, standardized, computer-assisted care-need assessment system. Level 1 refers to the group with the highest care needs, while Level 4 refers to the group with the lowest care needs. Level 5 refers to elderly who have dementia with relatively light functional limitations [3]. The care-need level data were checked from resident records and collected by staff nurses and/or social workers.

Nursing home staffing characteristics. Nursing home staffing characteristics included the use of RNs, number of nursing staff per bed, number of care workers per bed, and number of social workers per bed. Nursing staff included RNs and NAs. We also assessed whether nursing homes met the staffing standard. Following the Elderly Welfare Act 22 [6], we categorized nursing homes into two groups: yes=they met the standard, and no=they did not meet the standard. We present these staffing levels using their median values.

\section{Data Analysis}

The research team performed quality control and logic checks for the collected data. Descriptive statistics were used to describe the characteristics of the nursing homes, resident characteristics, their care needs, and care-provider staffing. By nursing home size, we examined differences in nursing homes' characteristics and residents' characteristics using $x^{2}$ tests and analysis of variance (ANOVA). Care-provider staffing levels were also compared by nursing home size using $x^{2}$ tests and ANOVA. All data analyses were performed using SAS version 9.4.

\section{RESULTS}

Nursing home characteristics are summarized in Table 1. Nursing homes were categorized into small nursing homes $(20.7 \%)$, small-medium nursing homes $(21.7 \%)$, medium nursing homes $(23.9 \%)$, and large nursing homes $(33.7 \%)$. The majority (93.5\%) of nursing homes in Korea were private. Greater proportions of small (94.7\%) and small-medium $(100.0 \%)$ nursing homes were also private. Occupancy rates of these sampled nursing homes were relatively low. About $65.0 \%$ of the nursing homes reported below $60.0 \%$ occupancy rates when the survey was conducted. Occupancy rates were not different by nursing home size. About half of the nursing homes were established after the LTCI was introduced in 2008. Smaller nursing homes (small: 63.2\%; small-medium: 75.0\%) tended to be established after the LTCI was introduced. Less than one-fourth (22.8\%) were located in rural areas. A higher proportion of medium nursing homes (40.9\%) were located in rural areas, although the proportion of small nursing homes in rural areas was not significantly different from that of medium nursing homes.

In terms of resident characteristics (Table 2), the mean age of residents was 82.7 (standard deviation: 7.48 ) years. The majority of nursing home residents were female (77.4 $\%$ ). About $26.0 \%$ of them were Medical-aid beneficiaries. Medium and large nursing homes had significantly higher proportions of residents who were Medical-aid beneficiaries, $31.3 \%$ and $28.8 \%$ respectively, compared to small (10.9 $\%)$ and small-medium (21.7\%) nursing homes. More than half $(67.9 \%)$ of the residents lived alone prior to admission.

Table 1. Characteristics of Nursing Homes

$(N=92)$

\begin{tabular}{|c|c|c|c|c|c|c|c|}
\hline \multirow{3}{*}{ Variables } & \multirow{3}{*}{ Categories } & \multirow[b]{2}{*}{ Total } & \multicolumn{4}{|c|}{ Nursing home size } & \multirow{3}{*}{$p$} \\
\hline & & & $\begin{array}{c}\text { Small } \\
(10 \sim 25 \text { beds })\end{array}$ & $\begin{array}{c}\text { Small-medium } \\
\text { (26 37 beds) }\end{array}$ & $\begin{array}{c}\text { Medium } \\
(38 \sim 62 \text { beds })\end{array}$ & $\begin{array}{c}\text { Large } \\
\text { (63 or more) }\end{array}$ & \\
\hline & & $\mathrm{n}(\%)$ & $\mathrm{n}(\%)$ & $\mathrm{n}(\%)$ & $\mathrm{n}(\%)$ & $\mathrm{n}(\%)$ & \\
\hline Total & & $92(100.0)$ & $19(20.7)$ & $20(21.7)$ & $22(23.9)$ & $31(33.7)$ & \\
\hline Ownership & $\begin{array}{l}\text { Private } \\
\text { Public }\end{array}$ & $\begin{array}{c}86(93.5) \\
6(6.5)\end{array}$ & $\begin{array}{c}18(94.7) \\
1(5.3)\end{array}$ & $\begin{array}{c}20(100.0) \\
0(0.0)\end{array}$ & $\begin{array}{r}19(86.4) \\
3(13.6)\end{array}$ & $\begin{array}{c}29(93.6) \\
2(6.4)\end{array}$ & .352 \\
\hline Occupancy rate (\%) & $\begin{array}{l}26.0 \sim 48.3 \\
48.4 \sim 58.4 \\
58.5 \sim 110.0\end{array}$ & $\begin{array}{l}32(34.8) \\
29(31.5) \\
31(33.7)\end{array}$ & $\begin{array}{r}5(26.3) \\
3(15.8) \\
11(57.9)\end{array}$ & $\begin{array}{l}7(35.0) \\
7(35.0) \\
6(30.0)\end{array}$ & $\begin{array}{l}9 \text { (40.9) } \\
8(36.4) \\
5 \text { (22.7) }\end{array}$ & $\begin{array}{r}11(35.5) \\
11(35.5) \\
9(29.0)\end{array}$ & .331 \\
\hline Year of foundation & $\begin{array}{l}2008 \\
\text { 2009 2011 }\end{array}$ & $\begin{array}{l}47(51.1) \\
45(48.9)\end{array}$ & $\begin{array}{r}7(36.8) \\
12(63.2)\end{array}$ & $\begin{array}{r}5(25.0) \\
15(75.0)\end{array}$ & $\begin{array}{l}12(54.6) \\
10(45.4)\end{array}$ & $\begin{array}{r}23(74.2) \\
8(25.8)\end{array}$ & .003 \\
\hline Location & $\begin{array}{l}\text { Rural } \\
\text { Urban }\end{array}$ & $\begin{array}{l}21(22.8) \\
71(77.2)\end{array}$ & $\begin{array}{r}2(10.5) \\
17(89.5)\end{array}$ & $\begin{array}{r}4(20.0) \\
16(80.0)\end{array}$ & $\begin{array}{r}9(40.9) \\
13(59.1)\end{array}$ & $\begin{array}{r}6(19.3) \\
25(80.7)\end{array}$ & .111 \\
\hline
\end{tabular}


Table 2. Characteristics and Care Needs of Residents in Nursing Homes

$(N=1,472)$

\begin{tabular}{|c|c|c|c|c|c|c|}
\hline \multirow{3}{*}{ Variables } & \multirow[b]{2}{*}{ Total } & \multicolumn{4}{|c|}{ Nursing home size } & \multirow{3}{*}{$p$} \\
\hline & & $\begin{array}{c}\text { Small } \\
\text { (10 25 beds) }\end{array}$ & $\begin{array}{l}\text { Small-medium } \\
\text { (26 37 beds) }\end{array}$ & $\begin{array}{c}\text { Medium } \\
\text { (38 62 beds) }\end{array}$ & $\begin{array}{c}\text { Large } \\
\text { (63 or more) }\end{array}$ & \\
\hline & $\begin{array}{l}\mathrm{n}(\%) \text { or } \\
\mathrm{M} \pm \mathrm{SD}\end{array}$ & $\begin{array}{l}n(\%) \text { or } \\
M \pm S D\end{array}$ & $\begin{array}{l}\mathrm{n}(\%) \text { or } \\
\mathrm{M} \pm \mathrm{SD}\end{array}$ & $\begin{array}{l}\mathrm{n}(\%) \text { or } \\
\mathrm{M} \pm \mathrm{SD}\end{array}$ & $\begin{array}{l}\mathrm{n}(\%) \text { or } \\
\mathrm{M} \pm \mathrm{SD}\end{array}$ & \\
\hline Total & $1,472(100.0)$ & $193(13.1)$ & $221(15.0)$ & $294(12.0)$ & 764 (51.9) & \\
\hline Age & $82.7 \pm 7.48$ & $82.4 \pm 7.39$ & $82.9 \pm 7.59$ & $83.1 \pm 7.48$ & $82.6 \pm 7.49$ & .735 \\
\hline $\begin{array}{l}\text { Gender } \\
\text { Male } \\
\text { Female }\end{array}$ & $\begin{array}{c}332(22.6) \\
1140(77.4)\end{array}$ & $\begin{array}{c}36(18.7) \\
157(81.3)\end{array}$ & $\begin{array}{c}46(20.8) \\
175(79.2)\end{array}$ & $\begin{array}{c}74(25.2) \\
220(74.8)\end{array}$ & $\begin{array}{l}176(23.0) \\
588(77.0)\end{array}$ & .345 \\
\hline $\begin{array}{l}\text { Medical-aid beneficiaries } \\
\text { Yes } \\
\text { No }\end{array}$ & $\begin{array}{c}381(25.9) \\
1091(74.1)\end{array}$ & $\begin{array}{c}21(10.9) \\
172(89.1)\end{array}$ & $\begin{array}{c}48(21.7) \\
173(78.3)\end{array}$ & $\begin{array}{c}92(31.3) \\
202(68.7)\end{array}$ & $\begin{array}{l}220(28.8) \\
544(71.2)\end{array}$ & $<.001$ \\
\hline $\begin{array}{l}\text { Living alone prior to admission } \\
\text { Yes } \\
\text { No }\end{array}$ & $\begin{array}{l}473(32.1) \\
999(67.9)\end{array}$ & $\begin{array}{c}46(23.8) \\
147(76.2)\end{array}$ & $\begin{array}{c}67(30.3) \\
154(69.7)\end{array}$ & $\begin{array}{l}119(40.5) \\
175(59.5)\end{array}$ & $\begin{array}{l}241(31.5) \\
523(68.5)\end{array}$ & .001 \\
\hline $\begin{array}{l}\text { Length of stay (days) } \\
\quad \leq 692 \\
\quad>692\end{array}$ & $\begin{array}{l}963.12 \pm 908.50 \\
736(50.0) \\
736(50.0)\end{array}$ & $\begin{array}{l}639.00 \pm 544.41 \\
118(61.1) \\
75(38.9)\end{array}$ & $\begin{array}{c}624.68 \pm 626.34 \\
146(66.1) \\
75(33.9)\end{array}$ & $\begin{array}{c}1014.23 \pm 973.49 \\
144(49.0) \\
150(51.0)\end{array}$ & $\begin{array}{l}1124.51 \pm 976.19 \\
328(42.9) \\
436(57.1)\end{array}$ & $\begin{array}{l}<.001 \\
<.001\end{array}$ \\
\hline $\begin{array}{l}\text { Stroke } \\
\text { Yes } \\
\text { No }\end{array}$ & $\begin{array}{c}411(27.9) \\
1061(72.1)\end{array}$ & $\begin{array}{c}39(20.2) \\
154(79.8)\end{array}$ & $\begin{array}{c}42(19.0) \\
179(81.0)\end{array}$ & $\begin{array}{c}72(24.5) \\
222(75.5)\end{array}$ & $\begin{array}{l}258(33.8) \\
506(66.2)\end{array}$ & $<.001$ \\
\hline $\begin{array}{l}\text { Dementia } \\
\text { Yes } \\
\text { No }\end{array}$ & $\begin{array}{l}886(60.2) \\
586(39.8)\end{array}$ & $\begin{array}{c}105(54.4) \\
88(45.6)\end{array}$ & $\begin{array}{c}127(57.5) \\
94(42.5)\end{array}$ & $\begin{array}{l}178(60.5) \\
116(39.5)\end{array}$ & $\begin{array}{l}476(62.3) \\
288(37.7)\end{array}$ & .185 \\
\hline $\begin{array}{l}\text { Activities of daily living } \\
\begin{array}{l}0 \sim 2=\text { Independent to limited } \\
\text { impairment } \\
3 \sim 4=\text { Extensive assistance } \\
\text { required } \\
5 \sim 6=\text { Dependent or totally } \\
\text { dependent }\end{array}\end{array}$ & $\begin{array}{l}420(28.5) \\
390(26.5) \\
662(45.0)\end{array}$ & $\begin{array}{l}49(25.4) \\
65(33.7) \\
79(40.9)\end{array}$ & $\begin{array}{l}53(24.0) \\
56(25.3) \\
112(50.7)\end{array}$ & $\begin{array}{l}101(34.4) \\
55(18.7) \\
138(46.9)\end{array}$ & $\begin{array}{l}217(28.4) \\
214(28.0) \\
333(43.6)\end{array}$ & .003 \\
\hline $\begin{array}{l}\text { Cognition } \\
\text { 0 2=Intact to mild } \\
\text { impairment } \\
3 \sim 4=\text { Moderate or severe } \\
\text { impairment } \\
5 \sim 6=\text { Severe or very severe } \\
\text { impairment }\end{array}$ & $\begin{array}{l}694(47.2) \\
420(28.5) \\
358(24.3)\end{array}$ & $\begin{array}{l}86(44.6) \\
55(28.5) \\
52(26.9)\end{array}$ & $\begin{array}{l}102(46.2) \\
67(30.3) \\
52(23.5)\end{array}$ & $\begin{array}{l}149(50.7) \\
86(29.3) \\
59(20.0)\end{array}$ & $\begin{array}{l}357(46.7) \\
212(27.8) \\
195(25.5)\end{array}$ & .565 \\
\hline $\begin{array}{l}\text { Depression } \\
\qquad \begin{array}{l}0=\text { Normal } \\
1 \sim 2=\text { Low risk } \\
3 \sim 14=\text { High risk }\end{array}\end{array}$ & $\begin{array}{l}471(32.0) \\
374(25.4) \\
627(42.6)\end{array}$ & $\begin{array}{l}63(32.7) \\
40(20.7) \\
90(46.6)\end{array}$ & $\begin{array}{l}76(34.4) \\
58(26.2) \\
87(39.4)\end{array}$ & $\begin{array}{l}108(36.7) \\
67(22.8) \\
119(40.5)\end{array}$ & $\begin{array}{l}224(29.3) \\
209(27.4) \\
331(43.3)\end{array}$ & .145 \\
\hline $\begin{array}{l}\text { Care-need level } \\
\text { Level } 1 \\
\text { Level } 2 \\
\text { Level } 3 \text { \& } 4\end{array}$ & $\begin{array}{l}205(13.9) \\
436(29.6) \\
831(56.5)\end{array}$ & $\begin{array}{c}18(9.3) \\
64(33.2) \\
111(57.5)\end{array}$ & $\begin{array}{c}23(10.4) \\
59(26.7) \\
139(62.9)\end{array}$ & $\begin{array}{c}28(9.5) \\
95(32.3) \\
171(58.2)\end{array}$ & $\begin{array}{l}136(17.8) \\
218(28.5) \\
410(53.7)\end{array}$ & .001 \\
\hline
\end{tabular}


A larger proportion (63.9\%) of these residents was admitted into medium and large nursing homes than into small (13.1\%) or small-medium (15.0\%) nursing homes. The average length of stay was 963.12 days (2.68 years). The length of stay of residents in small (639.00 days) or small-medium (624.68 days) nursing homes was significantly shorter than in medium (1,014.23 days) or large (1,124.51 days) nursing homes.

About $28.0 \%$ of residents were diagnosed with stroke while $60.2 \%$ of residents were diagnosed with dementia. Residents in larger nursing homes were more likely have had a stroke. In terms of $\mathrm{ADL}, 26.5 \%$ residents required extensive assistance while $45.0 \%$ of residents were dependent/totally dependent. Residents admitted into small nursing homes were less likely to require such assistance. More than half (52.8\%) of the residents had cognitive impairment above moderate levels. About $42.0 \%$ had a high risk of depression. These two conditions did not differ among residents by nursing home size. According to care-need level based on admission policy, $13.9 \%$ had Level 1 (the most complex conditions) while $29.6 \%$ had Level 2. Residents with Level 1 care-need levels admitted into large nursing home had a greater proportion (17.8\%) compared to residents admitted into other size nursing homes.

As for nursing home staffing (Table 3), less than half of the nursing homes hired at least one or more registered nurses (42.4\%). A greater proportion (77.4\%) of large nursing homes used RNs. Small-medium nursing homes were more likely to report lower levels of nursing staff adjusted by the number of beds. With regard to the legal nurse staffing requirement, $84.8 \%$ of nursing homes met this requirement. Both small and small-medium nursing homes met this requirement $(100.0 \%)$. In terms of care-worker staffing levels, small and small-medium nursing homes reported greater levels of care-worker staffing, although the difference in care-worker staffing levels between small/small medium nursing homes and medium/large nursing homes was not statistically significant. Only $37.0 \%$ of nursing homes overall met the legal care-worker staffing requirement when the survey was conducted. This did not significantly differ by nursing home size. Regarding social worker staffing levels, greater proportions of small (79.0\%) and small-medium (65.0\%) nursing homes reported higher levels of social worker staffing and met the social worker staffing requirement without showing a statistically significant difference between the two.

\section{DISCUSSION}

In this study, we found that a greater number of small

Table 3. Staffing Characteristics of Nursing Homes

$(N=92)$

\begin{tabular}{|c|c|c|c|c|c|c|c|}
\hline \multirow{3}{*}{ Variables } & \multirow{3}{*}{ Categories } & \multirow[b]{2}{*}{ Total } & \multicolumn{4}{|c|}{ Nursing home size } & \multirow{3}{*}{$p$} \\
\hline & & & $\begin{array}{c}\text { Small } \\
(10 \sim 25 \text { beds })\end{array}$ & $\begin{array}{l}\text { Small-medium } \\
\text { (26 37 beds) }\end{array}$ & $\begin{array}{l}\text { Medium } \\
\text { (38 62 beds) }\end{array}$ & $\begin{array}{c}\text { Large } \\
\text { (63 or more) }\end{array}$ & \\
\hline & & $\mathrm{n}(\%)$ & $\mathrm{n}(\%)$ & $\mathrm{n}(\%)$ & $\mathrm{n}(\%)$ & $\mathrm{n}(\%)$ & \\
\hline Total & & $92(100.0)$ & $19(20.7)$ & $20(21.7)$ & $22(23.9)$ & 31 (33.7) & \\
\hline Use of registered nurses & $\begin{array}{l}\text { Yes } \\
\text { No }\end{array}$ & $\begin{array}{l}39(42.4) \\
53(57.6)\end{array}$ & $\begin{array}{r}3(15.8) \\
16(84.2)\end{array}$ & $\begin{array}{r}4(20.0) \\
16(80.0)\end{array}$ & $\begin{array}{r}8(36.4) \\
14(63.6)\end{array}$ & $\begin{array}{r}24(77.4) \\
7(22.6)\end{array}$ & $<.001$ \\
\hline Nursing staff per bed & $\begin{array}{l}\leq 0.040 \\
>0.040\end{array}$ & $\begin{array}{l}47(51.1) \\
45(48.9)\end{array}$ & $\begin{array}{c}0(0.0) \\
19(100.0)\end{array}$ & $\begin{array}{r}18(90.0) \\
2(10.0)\end{array}$ & $\begin{array}{r}14(63.6) \\
8(36.4)\end{array}$ & $\begin{array}{l}15(48.4) \\
16(51.6)\end{array}$ & $<.001$ \\
\hline $\begin{array}{l}\text { Meeting nurse staffing } \\
\text { requirement }\end{array}$ & $\begin{array}{l}\text { Yes } \\
\text { No }\end{array}$ & $\begin{array}{l}78(84.8) \\
14(15.2)\end{array}$ & $\begin{array}{c}19(100.0) \\
0(0.0)\end{array}$ & $\begin{array}{c}20(100.0) \\
0(0.0)\end{array}$ & $\begin{array}{r}16(72.7) \\
6(27.3)\end{array}$ & $\begin{array}{r}23(74.2) \\
8(25.8)\end{array}$ & .007 \\
\hline Care workers per bed & $\begin{array}{l}\leq 0.369 \\
>0.369\end{array}$ & $\begin{array}{l}46(50.0) \\
46(50.0)\end{array}$ & $\begin{array}{r}8(42.1) \\
11(57.9)\end{array}$ & $\begin{array}{r}7(35.0) \\
13(65.0)\end{array}$ & $\begin{array}{l}11(50.0) \\
11(50.0)\end{array}$ & $\begin{array}{l}20(64.5) \\
11(35.5)\end{array}$ & .180 \\
\hline $\begin{array}{l}\text { Meeting care-worker } \\
\text { staffing requirement }\end{array}$ & $\begin{array}{l}\text { Yes } \\
\text { No }\end{array}$ & $\begin{array}{l}34(37.0) \\
58(63.0)\end{array}$ & $\begin{array}{r}11(57.9) \\
8(42.1)\end{array}$ & $\begin{array}{r}9(45.0) \\
11(55.0)\end{array}$ & $\begin{array}{r}7(31.8) \\
15(68.2)\end{array}$ & $\begin{array}{r}7(22.6) \\
24(77.4)\end{array}$ & .068 \\
\hline Social workers per bed & $\begin{array}{l}\leq 0.022 \\
>0.022\end{array}$ & $\begin{array}{l}46(50.0) \\
46(50.0)\end{array}$ & $\begin{array}{r}4(21.0) \\
15(79.0)\end{array}$ & $\begin{array}{r}7(35.0) \\
13(65.0)\end{array}$ & $\begin{array}{r}15(68.2) \\
7(31.8)\end{array}$ & $\begin{array}{l}20(64.5) \\
11(35.5)\end{array}$ & .003 \\
\hline $\begin{array}{c}\text { Meeting social worker } \\
\text { staffing requirement }\end{array}$ & $\begin{array}{l}\text { Yes } \\
\text { No }\end{array}$ & $\begin{array}{l}56(60.9) \\
36(39.1)\end{array}$ & $\begin{array}{r}15(79.0) \\
4(21.0)\end{array}$ & $\begin{array}{r}13(65.0) \\
7(35.0)\end{array}$ & $\begin{array}{r}13(59.1) \\
9(40.9)\end{array}$ & $\begin{array}{l}15(48.4) \\
16(51.6)\end{array}$ & .187 \\
\hline
\end{tabular}


and small-medium nursing homes were newly opened since the introduction of the public long-term care insurance (LTCI) in 2008. This is because long-term care is publicly funded. However, it is provided mostly by private nursing homes and home-care agencies in South Korea. Before the introduction of the public LTCI, only a limited number of nursing homes existed, most of which served economically disadvantaged older people, and their services were fully covered by taxes. Thus, the Korean government rapidly expanded the infrastructure for care provision at the inception of the pubic LTCI. It also encouraged private providers to enter into the LTC market, many of which tended to open small-sized nursing homes in urban areas due to limited budgets and concerns about the uncertainty of the new market. On the other hand, a greater proportion of medium nursing homes were located in rural areas. Their residents were more likely to be Medical-aid beneficiaries who lived alone prior to admission. Nursing homes located in rural areas might provide LTC services for older adults who have difficulty accessing care and lower demands for care.

An interesting finding of this study was that residents' characteristics and their care needs in Korean nursing homes were different by nursing home size. A smaller portion of residents in small-sized nursing homes were Medical-aid beneficiaries (10.9\%) or lived alone (23.8\%). On the other hand, medium nursing homes provided more care to Medical-aid beneficiaries $(31.3 \%)$ and residents who lived alone prior to admission (40.5\%). This indicates that medium nursing homes might play a role as safety-net hospitals for residents with limited economic resources and/or social support. The length of stay for residents in medium nursing homes was significantly longer than those in small and small-medium nursing homes. This finding suggests that medium nursing homes could experience financial hardship because they provide LTC services and social support for long time periods to residents with limited financial resources. Thus, the government should examine what support may need for these medium nursing homes to improve their staffing level and care quality.

Additionally, more than $20 \%$ of nursing home residents had suffered a stroke. This proportion was different by nursing home size. Residents in large nursing homes were more likely to have a stroke. This finding implies that residents in large nursing home may need more healthcare services rather than social services compared to residents in other sizes of nursing home. In this case, the appropriate staffing level of nurses and other health care professionals should be carefully examined, and systems for referring residents to acute-care hospitals also should be actively operated. Under the second national long-term care plan for 2018 2022, the Korean government plans to introduce into nursing homes special nursing units reimbursed by the public LTCI in order to strengthen health care for residents with complex dementia conditions [13]. The impact of such special units on the health outcomes of residents should be monitored and evaluated. In terms of ADL, more than $40 \%$ of nursing home residents were dependent or totally dependent. This was also different by nursing home size. This suggests that care workers who mainly provide ADL services are more frequently needed in small-medium and medium nursing homes. More than $20 \%$ of residents had severe or very severe cognitive impairments while more than $40 \%$ of residents had a high risk of depression. This suggests there is a great need for depression management among residents in nursing homes in Korea.

In terms of the staffing characteristics of nursing homes, small nursing homes were less likely to use RNs, although they met the legal nurse staffing requirement. In medium nursing homes, relatively fewer RNs were hired, and a lower proportion of those nursing homes met the nurse staffing requirement. Large nursing homes hired more RNs. With their lower staffing levels, as discussed above, medium nursing homes provide services to residents with lower resources (Medical-aid recipients and people living alone prior to admission). Based on this finding, there is a possibility that quality of care in medium nursing homes might be lower than in others. That may cause other disparity issues in nursing home care quality for these socioeconomically disadvantaged people. Thus, we need to further examine factors contributing to nursing home staffing and the impact of staffing levels on patient outcomes while adjusting for care needs, particularly for medium nursing homes.

The majority of nursing homes met the nurse staffing requirement. This does not mean those homes have an adequate staffing level. Rather, this is partly because the minimum staffing requirement is low for nursing homes and social care institutions under the public LTCI. As mentioned before, Korean nursing homes under the public LTCI were mainly designed as social-care modelbased homes. For this reason, compared to other countries, the nurse staffing requirement is relatively low. However, considering the complex health conditions of older residents presented in this study, it is suggested that there is a need to increase the staffing-level requirements overall. Other countries have also improved their 
minimum nurse-staffing standards. Evidence suggests this change has adjusted resident acuity and improved quality of care in nursing homes [14]. The Korean Ministry of Health and Welfare has also proposed a new staffing standard to meet residents' care needs in nursing homes, although it has not been approved yet [13]. In the ministry's proposal, nursing homes are required to hire more care workers and RNs.

Based on the study findings, there are several nursing implications. Residents' care needs and nursing home staffing levels were different by nursing home size. Characteristics of nursing homes and residents also varied by nursing home size. Although the current staffing standards are provided to meet minimum care-need requirements, residents' care needs vary by nursing home size. Thus, it is possible that some nursing homes may provide services that do not meet residents' care needs, which can lead to quality concerns. An advanced staffing standard considering case-mix should be implemented, for which a better monitoring of residents' care needs and nursing home staffing are necessary. This study has several limitations in that it was conducted with data collected in 2013. Since 2013, there may have been changes in residents' care needs and nurse staffing in the nursing homes. The study findings might not present the current status of residents' care needs and nurse staffing. However, the population representative study used here can provide figures related to national long-term care services, along with detailed care needs and staffing data by nursing home size; we are not aware of more recent, comparable data for Korean nursing homes under the public LTCI. According to nursing home size, residents' care needs, nursing home characteristics, and nurse staffing were quite different from each other. To an extent, we found disparity issues in care provision and staffing. This finding supports the significance of this study and presents a need for further research on this topic. In this study a cross-sectional study design was used which does not allow for identifying a cause-effect relationship. Thus, the interpretation of study findings should be conducted with caution. Family need, rather than residents' health conditions, is often an important factor in decisions to admit residents to nursing homes. In this study, we are not able to include this factor in the analysis. We also suggest further study is needed to collect data about residents' care needs and nurse staffing longitudinally and also include potentially missed variables. In this study, we found that only $37.0 \%$ of nursing homes met the care-worker staffing standard. Further studies are needed to find factors related to this under-staffing of care workers and its impacts.

\section{CONCLUSION}

South Korea has a policy agenda to increase nursing staffing level, especially registered nurses, and decrease variations in staffing levels by nursing home size. Along with such direct policy interventions for imminent challenges related to complex care needs and lower staffing requirements, a more fundamental approach might be needed, such as proper allocation of health-care resources based on care needs in both acute care settings (medical services) and long-term care settings (social services). Additionally, the government might need to allocate resources by regulation and implement policies to sustain the LTC system. Evidence on care-need levels and policy design are needed to strengthen the LTC workforce. This is essential to advance the LTC system in Korea.

\section{CONFLICTS OF INTEREST}

The authors declared no conflict of interest.

\section{AUTHORSHIP}

Study conception and design acquisition - BS-H and KH; Data collection - KH; Analysis and interpretation of the data - BS-H and $\mathrm{KH}$; Drafting and critical revision of the manuscript - BS-H and $\mathrm{KH}$.

\section{ACKNOWLEDGEMENT}

This research was supported by a grant of the Korea Health Technology R \& D Project through the Korea Health Industry Development Institute (KHIDI), funded by the Ministry of Health \& Welfare, Republic of Korea (Grant number: HI13C2250) and the AXA Research Fund 2016 AXA Award (No. 900-2017006) to the corresponding author. The funding source had no role in the study design; in the collection, analysis, and interpretation of data; in the writing of the manuscript; or in the decision to submit the manuscript for publication.

\section{REFERENCES}

1. United Nations, Department of Economic and Social Affairs, Population Division. World Population Ageing 2015 [Internet]. New York (NY): United Nations; 2015 [updated 2015 Jan 1; cited 2019 Sep 18]. Available from:

http://www.un.org/en/development/desa/population/pub lications/pdf/ageing/WPA2015_Report.pdf

2. Kinsella K, He W, U.S. Census Bureau. An Aging World: 2008 [Internet]. Washingon (DC): U.S. Government Prinitng Office; 2009 Jun 1 [updated 2009 Jun 1; cited 2019 Sep 19]. Available from: https://www.census.gov/prod/2009pubs/p95-09-1.pdf

3. National Health Insurance Service. Long-term care insurance 
statistics 2016 [Internet]. Wonju: National Health Insurance Services; 2017 [updated 2017 Jul 18; cited 2019 Sep 19]. Available from:

http://www.nhis.or.kr/bbs7/boards/B0160/24105?boardK ey $=37 \&$ sort $=$ sequence\&order $=$ desc\&rows $=10 \&$ messageCate goryKey $=\&$ pageNumber $=1 \&$ viewType=generic\&targetType $=12 \&$ targetKey $=37 \&$ status $=\&$ period $=\&$ startd $t=\&$ endd $t=\& q u$ eryField=\&query

4. Kim H, Jung YI, Kwon S. Delivery of institutional long-term care under two social insurances: lessons from the Korean experience. Health Policy. 2015;119(10):1330-7. https://doi.org/10.1016/j.healthpol.2015.07.009

5. Lee HY, Shin JH, Harrington C. Comparing the nurse staffing in Korean and U.S. nursing homes. Nursing Outlook. 2015;63 (2):137-43. https://doi.org/10.1016/j.outlook.2014.08.005

6. Ministry of Health and Welfare. Elderly Welfare Act 22 [Internet]. Sejong: National Law Information Center; 2018 [updated 2019 Sep 27; cited 2019 Sep 30]. Available from:

http://www.law.go.kr/lsBylInfoPLinkR.do?lsiSeq=184542\& lsNm=\%EB\%85\%B8\%EC\%9D\%B8\%EB\%B3\%B5\%EC\%A7\%8 0\%EB $\%$ B2\%95\%EC\%8B\%9C\%ED\%96\%89\%EA\%B7\%9C\%EC $\% \mathrm{~B} 9 \% 99 \&$ bylNo=0004\&bylBrNo=00\&bylCls=BE\&bylEfYd= \&bylEfYdYn=Y

7. Harrington C, Choiniere J, Goldmann M, Jacobsen FF, Lloyd L, McGregor M, et al. Nursing home staffing standards and staffing levels in six countries. Journal of Nursing Scholarship. 2012;44(1):88-98. https://doi.org/10.1111/j.1547-5069.2011.01430.x

8. Lee $\mathrm{Y}$, Jeong H, Seok JE, Song H, Seo D-M, Lee J, et al. A study of long-term care services planning [Internet]. Sejong: Ministry of Health and Welfare; 2017 Nov 1 [updated 2017 Nov 1; cited 2019 Nov 11] Available from: http://www.prism.go.kr/homepage/researchCommon/do
wnloadResearchAttachFile.do?work_key $=001 \&$ file_type $=C P$ R\&seq_no=001\&research_id=1351000-201800104\&pdf_conv -yn=N

9. Kim H, Jung YI, Sung M, Lee JY, Yoon JY, Yoon JL. Reliability of the interRAI Long Term Care Facilities (LTCF) and interRAI Home Care (HC). Geriatrics \& Gerontology International. 2015; 15(2):220-8. https://doi.org/10.1111/ggi.12330

10. Morris JN, Fries BE, Morris SA. Scaling ADLs within the MDS. The Journals of Gerontology. 1999;54(11):M546-53. https://doi.org/10.1093/gerona/54.11.M546

11. Morris JN, Fries BE, Mehr DR, Hawes C, Phillips C, Mor V, et al. MDS cognitive performance scale $\bigcirc$. Journal of Gerontology. 1994;49(4):M174-82. https://doi.org/10.1093/geronj/49.4.M174

12. Burrows AB, Morris JN, Simon SE, Hirdes JP, Phillips C. Development of a minimum data set-based depression rating scale for use in nursing homes. Age and Ageing. 2000;29(2): 165-72. https://doi.org/10.1093/ageing/29.2.165

13. Ministry of Health and Welfare. The 2018-2022 second stage of national plan of long-term care service in Korea [Internet]. Sejong: Misnistry of Health and Welfare; 2018 Jan 1 [updated 2018 Jan 1; cited 2019 Sep 19]. Available from:

http://www.google.com/url?sa=t\&rct=j\&q=\&esrc=s\&sourc e=web\&cd=1\&ved=0ahUKEwjm8NT9q6DbAhVGJpQKHZ mVBuUQFggnMAA\&url=http\%3A\%2F\%2Fwww.mohw.go. $\mathrm{kr} \% 2$ Freact\%2Fmodules\%2Fdownload.jsp\%3FBOARD_ID\% 3D140\%26CONT_SEQ\%3D343927\%26FILE_SEQ\%3D227707 \&usg=AOvVaw14Okr8ovMGdNugKiYpTeis

14. Harrington C, Schnelle JF, McGregor M, Simmons SF. The need for higher minimum staffing standards in U.S. nursing homes. Health Services Insights. 2016;9:13-9.

https:// doi.org/10.4137/HSI.S38994 